\title{
Questes
}

Revue pluridisciplinaire d'études médiévales

Journée d'étude 1 - Trier, classer, organiser | 2022

Trier, classer, organiser

\section{Voir la confusion, peindre l'ordre : le lieu sauvage de Leon Battista Alberti à Giovanni Bellini}

\section{Marie Piccoli-Wentzo}

\section{(2) OpenEdition}

1 Journals

Édition électronique

URL : https://journals.openedition.org/questes/5955

DOI : 10.4000/questes.5955

ISSN : 2109-9472

Éditeur

Les Amis de Questes

Édition imprimée

Pagination : 69-84

ISSN : 2102-7188

\section{Référence électronique}

Marie Piccoli-Wentzo, « Voir la confusion, peindre l'ordre : le lieu sauvage de Leon Battista Alberti à Giovanni Bellini », Questes [En ligne], Journée d'étude 1 - Trier, classer, organiser | 2022, mis en ligne le 31 janvier 2022, consulté le 21 février 2022. URL : http://journals.openedition.org/questes/5955 ; DOI : https://doi.org/10.4000/questes.5955 


\title{
Voir la confusion, peindre l'ordre : le lieu sauvage de Leon Battista Alberti à Giovanni Bellini
}

\author{
Marie PICCOLI-Wentzo \\ Université Paris 1 - Panthéon-Sorbonne
}

La perspective mathématique est essentielle à la représentation des lieux et des espaces en trois dimensions dans la théorie picturale en Italie $\mathrm{au} \mathrm{XV}^{\mathrm{e}}$ siècle. Pourtant, elle ne peut régir les formes de la nature sauvage : alors, comment représenter cette dernière ${ }^{1}$ ? À cette question, certains peintres répondent par un système d'exclusion de sorte que les terres sauvages sont dépeintes en réaction aux espaces civilisés et ainsi, au sein du même cadre, tous les espaces sont peints: de la ville avec ses murailles aux pics courbés des montagnes indomptables. Cet antagonisme donne naissance à un espace qu'on peut parcourir. Ainsi, pour figurer un lieu sauvage, les peintres représentent un système de zones régenté selon l'organisation de la société médiévale. Plus précisément, l'espace peint répond à une mise en ordre du monde dans laquelle le lieu inhospitalier s'apparente à une «structure d'exclusion ${ }^{2}$ ». Hypothétiquement, nous pourrions penser que peindre la nature sauvage équivaudrait donc à

\footnotetext{
${ }^{1}$ Cet article s'insère dans une étude plus vaste consacrée à l'image du désert dans la peinture de la Renaissance faisant l'objet d'une thèse de doctorat en préparation à l'Université Paris 1 Panthéon-Sorbonne.

${ }^{2}$ Je reprends ici l'expression d'Hubert Damisch quant au rapport entre le ciel, les nuages et la perspective ; expression reprise et adaptée par Daniel Arasse concernant la nature. Hubert Damisch, Théorie du nuage. Pour une histoire de la peinture, Paris, Seuil, 1972, p. 170-171 ; Daniel Arasse, «L'Espace de la perspective et le lieu du paysage », dans Leon Battista Alberti, Actes du Congrès International de Paris, 1015 avril 1995, t. II, Paris/Turin, Vrin/Nino Aragno, 2000, p. 588-597, p. 590-591.
} 
démontrer qu'elle est bien une terre marginale, tout autant qu'à exposer cette mise en ordre du monde par le truchement d'une représentation visuelle.

L'organisation de ces espaces, au sein des peintures italiennes du $\mathrm{XV}^{\mathrm{e}}$ siècle, trouve une résonance particulière dans les écrits du polymathe Leon Battista Alberti. Son traité de peinture ne dit presque rien de la représentation du paysage; en revanche, le théoricien présente, dans l'Art d'édifier, un monde organisé en trois territoires ${ }^{3}$. Trois zones concentriques régissent l'ensemble de l'espace italien : la cité est au cœur de la composition, elle est entourée par la regio c'est-à-dire la campagne, elle-même cernée par un ensemble plus vaste et non délimité, la provincia. Sur ce découpage pragmatique, Alberti associe à chaque zone une conception poétique ainsi que des notions plastiques suggérant une possible construction imagée. De la sorte, la cité est un lieu habitable répondant à l'intelligibilité, elle est un espace (spatium) clos, délimité par des murailles et construit à l'aide de lignes droites et géométriques; la perspective régit, par conséquent, l'aire de la ville. La regio est définie comme une extension de la ville, il s'agit de la nature soumise à la ville et donc, une nature domptée par l'homme. Elle est un lieu non ceint mais constituant un entre-deux, un intermédiaire entre deux pôles antagonistes. Enfin, l'auteur évoque la provincia comme un « reste $^{4} »$ dont il ne dit rien et qui se construit alors comme l'opposé de la ville : cette nature sauvage est donc un lieu déserté, un «intervalle sans figure ${ }^{5}$ », qui résiste à l'intelligibilité, elle est un espace sans limites et se trouve construite par

\footnotetext{
${ }^{3}$ Leon Battista Alberti, L'Art d'édifier [De Re Aedificatoria], I, 7, Paris, Seuil, 2004, p. 73-74. Pour l'analyse linguistique de ces passages, Daniel Arasse, «L'espace de la perspective et le lieu du paysage », art. cit., p. 588-597.

${ }^{4}$ Cf. Daniel Arasse, «L'Espace de la perspective et le lieu du paysage », art. cit., p. 592.

${ }^{5}$ Cf. Ibid. Pour la notion d'objet figuratif, Pierre Francastel, La Figure et le lieu, l'ordre visuel du Quattrocento, Paris, Gallimard, 1967, p. 63 et suiv.
} 
des serpentines et des spirales-lignes qui étaient exclues de la construction de la cité. Ce passage rédigé par l'érudit humaniste est fondamental pour la perception de la perspective et de ce qui se conçoit comme son opposé dans la théorie de l'art du Quattrocento puisque les deux projections plastiques y recueillent leur implication imaginaire. Le lieu, sa poétique et les moyens plastiques de sa représentation visuelle sont réunis par Alberti et on constate que chaque zone définit, au-delà de sa fonction pour l'homme, un tout constitutif de l'homme ${ }^{6}$.

J'aimerais ici étudier, à travers quelques exemples puisés dans la peinture italienne (Sienne, Florence et Venise) de la seconde moitié du $\mathrm{XV}^{\mathrm{e}}$ siècle et au début du $\mathrm{XVI}^{\mathrm{e}}$ siècle, le cadre qu'offre la peinture à la nature, et plus précisément lorsque cette dernière est située dans un contexte érémitique; en d'autres termes, de quelle manière la peinture organise-t-elle le monde extérieur ? Quels sont les moyens de cette mise en ordre ? Qui est l'ordonnateur de cette nature - l'homme, Dieu, l'artiste ou la nature elle-même ? Cette mise en ordre refléterait-elle une réflexion sur les origines : les temps de la Création divine ou, de manière réflexive, la trace d'un geste créateur laissé par l'artiste, un symptôme d'une création artistique ? Enfin, dans quelle mesure l'organisation de la nature

\footnotetext{
${ }^{6}$ Les écrits d'Alberti servent essentiellement à fixer une pensée déjà en cours au moment de son écriture. On trouve, probablement pour cette raison, des résonances du texte dans la peinture siennoise au moment même de sa composition entre 1442 et 1452. Que le texte ait été connu par les peintres ou que ces représentations découlent de l'innutrition, un lien entre société et imaginaire se tisse à travers le pouvoir accumulatif de l'art. Pour plus d'informations sur le rapport entre Alberti et les peintres de son époque: Maurice Brock, «La Phobie du "tumulte" dans le De pictura », Albertiana, vol. 8., 2005, p. 119-180, p. 157-159; Maurice Brock, «Entre mesurage et commensuration - II les dimensions relatives des personnages et des architectures dans quelques œuvres toscanes du XV siècle», Albertiana, vol. 20, 2017, p. 37-76, p. 38 et suiv. et plus particulièrement p. 41 ; Michael Baxandall, Giotto et les humanistes. La découverte de la composition en peinture 1340-1450, Paris, Seuil, 2013, p. 95-221 ; Daniel Arasse, «L'Espace de la perspective et le lieu du paysage », art. cit., p. 592-593.
} 
par la peinture est-elle au cœur de l'interaction entre l'image, l'imaginaire et l'imagination?

\section{Un geste ordonnateur : du vacuum au parergon}

Un panneau peint par Giovanni di Paolo et daté entre 1455 et 1460 constitue un des exemples les plus proches du texte albertien et permet de démarrer une réflexion sur nos interrogations ${ }^{7}$ (fig. 1). Dans l'espace conçu par Giovanni di Paolo, trois zones paysagères s'articulent : au bord inférieur gauche, la ville ou plus précisément, l'une des portes délimitant l'espace de la ville ; dans la partie inférieure droite, des champs rangés à l'intérieur d'une structure linéaire ; enfin dans la partie supérieure, des montagnes rocheuses dessinées par des lignes obliques et courbes. Déambulant dans cette construction paysagère improbable, le jeune saint Jean-Baptiste chemine d'un espace à un autre, en deux temps successifs : d'abord, sous l'encadrement de la porte de la cité, le garçonnet fait un pas vers l'extérieur, vers une première nature - celle soumise à l'homme, la regio -; puis, plus loin, sur le chemin menant au cour de la nature sauvage, il s'enfonce dans un désert de roches montagneuses. La figure du petit saint apparaît deux fois; lors de ces deux reprises, elle est démesurée, bien trop grande : d'abord dans l'encadrement de la porte, elle remplit plus de la moitié de la hauteur puis, dans le lointain, la taille du personnage n'a pas été diminuée - à l'inverse, les édifices érigés dans la campagne paraissent minuscules face à cet enfant saint ${ }^{8}$. Sa double

\footnotetext{
${ }^{7}$ Daniel Arasse note déjà dans son article la résonance entre le texte albertien et la peinture de Giovanni di Paolo, pour cela, il se sert du panneau du même thème, légèrement antérieur puisque daté de 1454 et aujourd'hui conservé à la National Gallery de Londres. Pour plus de détails, Daniel Arasse, «L'espace de la perspective et le lieu du paysage », art. cit.

${ }^{8}$ Sur le rapport de taille entre les personnages et les édifices chez Alberti : Maurice Brock, «Entre mesurage et commensuration. I Les dimensions relatives des
} 
présence établit la fonction du lieu qui peut être parcouru tandis que ce dernier instaure, en corollaire, un vide autour des figures permettant la lisibilité de la scène. Le lieu joue ainsi le rôle de vacuum, ce vide indispensable selon Alberti, pour permettre le déroulement de l'istoria ${ }^{9}$. Au-delà d'une organisation reflétant la société, le parcours rendu possible par la construction hétérogène de la nature renvoie à différentes temporalités et le vacuum permet l'organisation ainsi que la lecture visuelle de l'histoire contée.

Si le lieu comme vacuum relève d'un geste ordonnateur de la part du peintre, un geste poétique est révélé par un autre effet pictural, le parergon - c'est-à-dire ce qui est en marge de l'ergon, de l'œuvre. La nature répond effectivement à trois fonctions du parergon comme les renaissants l'ont redécouvert et réinterprété ${ }^{10}$ : celui-ci permet d'abord de distinguer les figures - ici, les temporalités de notre unique personnage ; il est aussi un accessoire de la figure - pour nous, la retraite de saint Jean-Baptiste -; enfin, et c'est la notion la plus importante qui se combine avec la précédente, il témoigne de l'imagination ainsi que de la réflexion de l'artiste et donne alors une vie organique aux choses qu'il revêt. À partir de cette observation, nous pouvons déduire que trois idées s'enchevêtrent sur le tissu sémantique que constitue le parergon: la construction et la dé-construction de la perspective ; la juxtaposition des

personnages et des architectures dans le De pictura », Albertiana, vol. 18, 2015, p. 5-56.

${ }^{9}$ Vacuum et istoria renvoient aux termes utilisés et définis par Alberti. Leon Battista Alberti, La Peinture [De Pictura], II, 40, Paris, Seuil, 2004, p. 141-143.

${ }^{10}$ Pour une étude du parergon à la Renaissance, Mathilde Bert, «La Genèse du parergon: usages renaissants d'un concept antique sur la peinture », dans $L a$ Mécanique du détail. Lectures transversales, dir. Livio Belloï et Maud Hagelstein, Lyon, Éditions ENS de Lyon, 2012, p. 141-154 ainsi que Pline l'Ancien, Histoire naturelle. Livre XXXV. La peinture, trad. J.-M. Croisille, Paris, Les Belles Lettres, 1985, §101 ; Strabon, Géographie, XIV, 2, 5, Paris, Macula, 1985, n 500, p. 373 ; Vitruve, De l'Architecture, Livre IX, trad. J. Soubiran, Paris, Les Belles Lettres, 1969, chap.8, §4-5. 
poétiques contraires de la rationalité et de l'inintelligible; enfin la contrainte organisatrice (vacuum) et l'imagination créatrice (parergon) dont l'artiste doit faire preuve. Une déconstruction progressive de la perspective semble d'abord se mettre à l'œuvre dans les lieux de l'image puisque la perspective linéaire construisant le bout de muraille du bord gauche s'aplanit dans le quadrillage des champs cultivés du bord droit avant de disparaître à l'entrée du monde sauvage. D'autres formes et par conséquent une autre construction remplacent alors cette première structure. Trois états successifs émergent par cette simple description : la construction perspective en trois dimensions, son aplanissement et la mise en place d'un quadrillage-qui pourrait rappeler les outils artistiques pour dompter la nature à travers l'art graphique - ; enfin, une zone de recréation plus libre. En ce sens, je pense que ces trois zones rejouent, au moins d'une certaine manière, une des célèbres métaphores de la peinture qui pour exister, passe d'un état solide (les pigments) à un état liquide (la matière fluide), pour redevenir solide (le support peint). Quoiqu'il en soit, l'entrée dans les terres du sauvage correspond à une pénétration dans le monde de l'imaginaire, au sein d'un lieu gouverné par la poétique de l'irrationnel, du merveilleux, du fantasme et de l'esprit. Ce lieu et sa forme font appel à l'imagination et à la réflexion de l'artiste, à sa fantasia $^{11}$. L'artiste peut ainsi donner forme à ce qui n'existe pas et, par là, prouver l'équivalence créatrice entre l'art de peindre et la poésie ${ }^{12}$.

\footnotetext{
${ }^{11}$ L'imagination et la fantasia ne sont pas toujours synonymes ; pour l'explication et la distinction des deux termes au Quattrocento : Martin Kemp, «From "Mimesis" to "Fantasia" - the Quattrocento Vocabulary of Creation, Inspiration and Genius in the Visual Arts », Viator, vol. 8, 1977, p. 347-398, p. 361-384.

12 Cennino Cennini considère que la « fantasia e hoperazione di mano, di trovare cose non vedute », c'est-à-dire que le peintre a la capacité de créer des formes qui n'existent pas dans la nature ; l'idée provient d'Horace, Cennino Cennini, Il libro dell'arte, I, 1, Vicence, Neri Pozza Editore, 2011, p. 62 ; Horace, Ars poetica 9-10 dans Q. Horatii Flacci Epistula ad Pisones de arte poetica: Horace, art poétique,
} 


\section{Déambulations spatiales et confusion plastique}

Dans une toile datée des environs de 1470 et peinte par Paolo Uccello, les trois zones sont à nouveau figurées ${ }^{13}$ (fig. 2). Au loin une cité ceinte de murailles blanches surplombe une nature cultivée par l'homme pour ses besoins alors qu'à l'avant une structure complexe se développe sur le schéma ascensionnel d'une montagne rocheuse-reprenant le dessin en courbe des pics, déjà observé chez Giovanni di Paolo. Dès lors, le lieu sauvage acquiert une structure élargie qui me permettra de préciser ma réflexion. L'ordre établi par la forme montagneuse est articulé par des lieux de mémoire. Ces loci sont délimités par des structures circulaires formées dans ou par la nature - creux dans la roche, disposition des arbres, arc-en-ciel, air donnant un mouvement aux nuages. Ces pauses sont organisées comme un parcours érémitique au cours duquel différentes activités dévotionnelles sont pratiquées ${ }^{14}$. Ainsi, de la base au faîte de l'édifice mnémonique, l'esprit du spectateur s'oriente vers une profonde méditation menant de la lecture de textes sacrés à la vision de Dieu. Mémoire et fantasia participent, dans la toile de l'Accademia, à la mise en ordre du monde représenté et fonctionnent comme des accessoires visuels du cheminement peint. En effet, la structure mnémonique de la montagne perforée laisse place à une déconstruction

texte latin, éd. Maurice Albert, Paris/Londres, Hachette, 1986 ; Martin Kemp, « From "Mimesis" to "Fantasia"... », art. cit., p. 368.

${ }_{13}$ Pour l'appareil critique de la peinture, Franco et Stefano Borsi, Paolo Uccello, Paris, Hazan, 1992, 2004, p. 335-336. Pour une synthèse plus récente: Andrea Staderini, «La Vita di perfezione attribuita a Paolo Uccello»dans Atlante delle Tebaidi e dei temi figurativi, dir. Alessandra Malquori, Manuela De Giorgi, Laura Fenelli, Firenze, Centro Di, 2013, p. 76-80.

${ }^{14}$ Pour plus d'informations sur les arts de la mémoire, Frances Yates, L'Art de la mémoire, Paris, Gallimard, 1975 ; Mary Carruthers, The Book of Memory: A Study of Memory in Medieval Culture, Cambridge, Cambridge University Press, 1992. Pour le parcours ascensionnel dans la toile de Paolo Uccello, Alessandra Malquori, Il giardino dell'anima: ascesi e propaganda nelle "Tebaidi" fiorentine del Quattrocento, Firenze, Centro Di, 2012, p. 27-29. 
plus forte de la perspective, sous l'apparence du ciel orageux et de sa nébuleuse. Or, mémoire et fantasia sont réunies dans les théories philosophiques de la Renaissance, au moins sous la plume de Marsile Ficin, puisque ce dernier en fait deux facultés issues de l'intelligence ${ }^{15}$. L'association semble bien trouver une illustration dans la peinture de Paolo Uccello. Alors que dans l'organisation de la société, le lieu sauvage apparaît négatif, dangereux et néfaste, il est, dans cette peinture glorifiant le mode de vie érémitique, un espace mystique, permettant l'accès au divin et à la perfection de l'image retrouvée de Dieu. Au plus fort de la déconstruction formelle, l'œil ne perçoit que le trouble et l'agitation d'une vue obstruée, autrement dit, d'une perspective inexistante tandis que l'esprit, l'intellect atteint un degré supérieur de clarté et de compréhension, comme s'il fallait, en corrélation avec les pratiques ascétiques, pousser ses limites - corporelles, visuelles, intellectuelles pour atteindre le point culminant, le summum de l'imagination créatrice.

La célèbre chapelle des Mages du Palazzo Medici-Ricardi à Florence offre un autre exemple non moins révélateur ${ }^{16}$. Le décor monumental représente la procession de la fête de l’Épiphanie qui était un des grands évènements de la ville toscane ${ }^{17}$. Tous les ans ou presque, pour commémorer la naissance du Christ, les Florentins rejouaient le fameux voyage des mages. La procession menait du Palazzo Vecchio,

\footnotetext{
${ }^{15}$ Marsile Ficin, Théologie platonicienne de l'immortalité des âmes, IX, 5 ; XI, 3 ; $\mathrm{XV}, 16$. Cité dans : Thierry Gontier, «Un platonisme sans cosmos ? La sagesse de Marsile Ficin»dans Marsile Ficin: les platonismes à la Renaissance, dir. Pierre Magnard, Paris, J. Vrin, 2001, p. 69-89, p. 77

${ }^{16}$ Pour 1'appareil critique du panneau : Jeffrey Ruda, Fra Filippo Lippi: Life and Work with a Complete Catalogue, London, Phaidon Press, Abridged edition, 1993, p. 447-448 et aussi p. 218-235; ainsi que Megan Holmes, Fra Filippo Lippi: The Carmelite Painter, New Haven/London, Yale University Press, 1999, p. 172-182.

${ }^{17}$ Il s'agit des célèbres fresques peintes par Benozzo Gozzoli en 1459. Pour plus d'informations sur les fresques, Diane Cole Ahl, Benozzo Gozzoli, Paris, Éditions du Regard, 1996, 2002, p. 84 et suiv. ; Cristina Acidini Luchinat, Benozzo Gozzoli : la cappella dei Magi, Milano, Electa, 1993, p. 19 et suiv.
} 
lieu du pouvoir administratif, au couvent San Marco, lieu de culte ${ }^{18}$. Les personnages importants de la ville, les Médicis en tête, présidaient la procession en tenant le rôle des mages. La chapelle est alors commémorative du récit biblique, de l'évènement florentin et de la place sociale de la famille dans la capitale toscane. Les fresques de la chapelle déclinent ce voyage par des chemins dessinés depuis les lointains, des foules rassemblées avançant et convergeant vers le même point : le retable de la chapelle au sein duquel une dense forêt rompt toute concordance paysagère avec les fresques. L'épaisseur de la végétation, l'absence du ciel et du soleil rappelleraient le massif forestier du Casentino, à quelques kilomètres de Florence $^{19}$; ce lieu mystique renferme le berceau de l'ordre des camaldules, ses ermites visionnaires, ses légendes miraculeuses et ses récits sacrés. C'est pourquoi cette forêt a grandement marqué l'imaginaire italien et Dante, tout particulièrement, s'en fait l'écho sous la forme d'un désert, dans sa Divine comédie ${ }^{20}$.

${ }^{18}$ Pour plus d'informations sur le théâtre sacré et la fête de l'Épiphanie à Florence, Sophie Stallini, Le Théâtre sacré à Florence au XVe siècle : une histoire sociale des formes, Paris, Presses Sorbonne nouvelle, 2011, p. 133.

${ }^{19}$ Pour plus d'informations sur le rapport entre la forêt peinte par Filippo Lippi et le massif forestier du Casentino, ainsi qu'entre la famille Médicis et l'ermitage des camaldules, Stefanie Solum, Women, Patronage, and Salvation in Renaissance Florence: Lucrezia Tornabuoni and the Chapel of the Medici Palace, Farnham, Ashgate, 2015, p. 109 et suiv. Pour plus d'informations sur l'ordre des camaldules, Cécile Caby, De l'érémitisme rural au monachisme urbain, les Camaldules en Italie à la fin du Moyen Âge, Rome, École française de Rome, 1999 ; Cécile Caby, Pierluigi Licciardello, Camaldoli e l'ordine camaldolese dalle origine alla fine del XV secolo: atti del I Convegno internazionale di studi in occasione del millenario di Camaldoli (1012-2012), monastero di Camaldoli, 31 maggio-2 giugno 2012, Cesena, Centro Storico Benedettio Italiano, 2014.

${ }^{20}$ D'autres auteurs ont comparé la peinture de Filippo Lippi au désert forestier de Dante, voir entre autres : Beatrice Paolozzi Strozzi, «The Adoration of the Child by the Workshop of Filippo Lippi », dans The Chapel of the Magi: Benozzo Gozzoli's Frescoes in the Palazzo Medici-Riccardi Florence, dir. Cristina Acidini-Luchinat, London, Thames and Hudson, 1994, p. 29-32 ; Dale Kent, Cosimo de' Medici and the Florentine Renaissance, New Haven/London, Yale University Press, 2000, p. 308-325 ; Patricia Rubin, «Signposts Of Invention, Artists' Signatures In Italian Renaissance Art. », Art History, vol. 29, no 4, 2006, p. 563-99, p. 573. À deux reprises, Dante use d'un désert sous forme forestière : l'auteur se perd, lors du 
Ainsi, trois pérégrinations se trouvent accumulées dans la chapelle des Mages : le voyage des mages vers Bethléem, la procession florentine lors de la fête de l'Épiphanie et la retraite des Médicis menant de leur palais urbain à l'ermitage des camaldules.

Or, la déambulation dans la chapelle entraîne le spectateur au cœur d'une véritable expérience visuelle. Un premier passage s'opère entre la clarté des fresques et l'obscurité de la forêt peinte par Filippo Lippi. Cet effet était, au milieu du $\mathrm{XV}^{\mathrm{e}}$ siècle, renforcé par le contexte d'exposition : seuls deux oculi permettaient de faire entrer les rayons du soleil dans la chapelle, si bien que l'espace devait, très probablement, être constamment éclairé à la lueur de bougies ${ }^{21}$. De cette manière, nous pouvons en déduire que lorsque le retable était dans son lieu de destination originelle, il devait être visuellement enrichi par un certain mysticisme: la lumière jaillissante entre les personnes divines partiellement, recouverte à la feuille d'or via une technique au mordant devait étinceler sous l'effet des bougies et ressortir comme une source de lumière au cœur des ombres de l'espace forestier ${ }^{22}$. Cet effet visuel, assurément saisissant, trouve une résonance dans le programme iconographique : à la sombre forêt où règne le désordre, s'oppose la

préambule, au cœur d'une forêt avant d'entamer son voyage visionnaire, ce lieu est une métaphore de la perte de soi ; puis, au sommet de la montagne du Purgatoire, le désert-forêt apparaît comme un vaste espace préambulatoire du Paradis. Le sujet de la peinture de Filippo Lippi relie, par les thèmes iconographiques représentés, ces deux significations symboliques. Pour une étude du désert chez Dante, Marcello Castellana, «Pour une sémiotique du désert : Dante, Divina Commedia, Purgatorio XI, 13-15 », dans Le Désert, un espace paradoxal, Actes du colloque de l'Université de Metz, 1315 septembre 2001, dir. Gérard Nauroy, Pierre Halen, Anne Spica, Bern/Berlin/Bruxelles, Peter Lang, 2003, p. 161-193.

${ }^{21}$ Pour les circonstances d'exposition, Dale Kent, Cosimo de' Medici and the Florentine Renaissance, op. cit., p. 308-325.

${ }^{22}$ Pour une étude de l'usage de l'or dans le retable, Rose Miller, Christine Patrick, «Four Weeks of Work for four Seconds of Fame: Reconstructions for Television », dans Lucy Wrapson, Jenny Rose, Rose Miller, Spike Bucklow (éd.), In Artists Footsteps, the Reconstruction of Pigments and Paintings, London, Archetype publications, 2012, p. 165-176, p. 170-172. 
rédemption de l'homme, figurée par la descente sur terre du Dieu incarné et qui plus est, cet épisode bénéficie d'une métaphore sur le recouvrement de la vue par l'homme qui reconnaît l'Incarnation ${ }^{23}$. En somme, cette réflexion sur la vision s'associe à une dialectique de l'ombre et de la lumière, renvoyant à une expérience, celle de la visibilité. Force est alors de constater que la confusion plastique renforce la faculté de voir, et plus précisément celle de bien voir. Au cours des trois exemples que nous avons analysés, la peinture se fait le support d'une réflexion, permettant de se perdre dans le but de se (re)trouver. Ainsi, l'image devient une métaphore du voyage de l'esprit projeté, à travers la surface picturale, dans un lieu mystique de méditation.

\section{De la surabondance de formes au débordement de la matière}

D'autres exemples nous permettent d'apprécier une solution différente: l'image double. Ainsi, dans le Saint Jérôme lisant de Giovanni Bellini conservé à la National Gallery of Art de Washington, la composition du lieu en trois zones reparaît avec quelques modifications : la ville entourée de murailles blanches dans les lointains ; un ensemble d'édifices en ruines; enfin une caverne, abritant la retraite de saint Jérôme. Cette régression architectonique met en scène un retour à la nature primitive à travers, d'une part, un mode de vie ascétique et austère pour le corps et, d'autre part, un état contemplatif et émerveillé pour l'esprit. Au sein de la nature sauvage, des images doubles dotent la roche d'une vie: au moins trois visages minéraux émergent de la cavité rocheuse derrière l'ascète. Directement au-dessus de la tête de saint Jérôme, un visage sculpté se distingue dans la roche et, en face de ce

\footnotetext{
${ }^{23}$ Pour plus d'informations sur le recouvrement de la vue par l'homme lors de la naissance christique, Michel Weemans, Herri Met de Bles : les ruses du paysage au temps de Bruegel et d'Érasme, Paris, Hazan, 2013, p. 104-133 et p. 226-267.
} 
dernier, deux combinaisons de pierres à valeur figurative forment deux inquiétants profils (fig. 3). D'autres formes plus discutables pourraient encore être au stade inchoatif. L'imagination n'est alors plus inhérente à l'artiste, elle passe par le regard du spectateur: dès lors, ces images exigent une participation active de la part du regardeur et appellent à l'observation et à l'interprétation subjectives ${ }^{24}$. Au-delà de tout sens dévotionnel ou symbolique, ces images doubles participent à un discours sur l'art. La déconstruction paysagère permet ici la construction de nouvelles formes. D'un point de vue humaniste, ces images traduisent l'idée antique «de la nature tiraillée entre l'art(tekhnè) et le hasard (tukhè) ${ }^{25}$ », mêlant l'habilité de la main de l'artiste à la spontanéité de la matière, à savoir la nature devenant elle-même artiste. Dans la peinture de Giovanni Bellini, l'ambiguïté visuelle proposée touche à l'art sculptural, entre techniques de la taille et du modelage.

Titien prend, presque cinquante ans plus tard, un autre chemin : probablement sous le couvert d'un autre effet pictural, celui du colorito, il donne à voir au spectateur un rapprochement entre la nature sauvage et la matière picturale libérée de toute forme figurative ${ }^{26}$. À la surabondance

\footnotetext{
24 À ce sujet, David Freedberg, Le Pouvoir des images, Paris, G. Monfort, 1998.

${ }^{25}$ Cf. Bertrand Prévost, «Andrea Mantegna, Triptyque de l'Adoration des mages », dans Voir double: pièges et révélations du visible, dir. Michel Weemans, Dario Gamboni, Jean-Hubert Martin, Paris, Hazan, 2016, p. 160. Pour plus d'informations, Michel Weemans, Herri Met de Bles : les ruses du paysage au temps de Bruegel et d'Érasme, op. cit.; Dario Gamboni, Images potentielles. Ambiguité et indétermination dans l'art moderne, Dijon, Les Presses du Réel, 1992, 2016 ; The Anthropomorphic Lens: Anthropomorphism, Microcosmism and Analogy in Early Modern Thought and Visual Arts, dir. Walter S. Melion, Breth L. Rothstein, Michel Weemans, Leiden/Boston, Brill, 2014.

${ }^{26}$ Pour un autre point de vue sur les coulures dans l'œuvre de Titien: Guillaume Cassegrain, La Coulure, histoire(s) de la peinture en mouvement : XI ${ }^{e}-X X I^{e}$ siècles, Paris, Hazan, 2015, p. 225-226 ; Guillaume Cassegrain, «Pleurer, voir. À propos d'un détail du saint Jean-Baptiste de Titien », Venezia Cinquecento, vol. 35 : «Il grande vecchio Tiziano, 1540-1576 », 2008, p. 139-154 ; Guillaume Cassegrain, «Titien, Saint Jean-Baptiste », dans Voir double: pièges et révélations du visible, op. cit., p. 202.
} 
de formes, Titien appose un excès de matière. Ainsi son Saint Jérôme conservé à l'Escurial dépeint une nature à la fois surprenante et extraordinaire, composée d'éruptions, de déversements, de saturation de la matière picturale ; une troublante pléthore picturale s'offre désormais à la vue inquiète de l'observateur.

En somme, si l'organisation de la nature permet une mise en ordre du monde, toutes deux sont traduites en peinture par un usage varié de moyens plastiques. D'une part, le peintre organise un cheminement à travers une déconstruction plastique qui renvoie à la mise en ordre structurelle du monde, tel qu'il est pensé par la société. De la sorte, les différents espaces peints font cheminer l'esprit du visiteur qui s'attarde un tant soit peu devant l'image, d'un monde plastique à un autre et en prolongement, d'un espace rationnel à un lieu imaginaire. En d'autres termes, la peinture supporte et renforce la capacité de penser, de méditer et d'imaginer de l'homme. Cet effort est soutenu par un rythme visuel formel organisé par le geste ordonnateur du peintre. D'autre part, le désert, lieu frontière de toute rationalité humaine, nourrit, tout à la fois, la réflexion, l'imagination, la fantasmagorie et, en somme, l'activité psychique de l'homme. C'est pourquoi les peintres, afin de mettre en exergue cette structure d'exclusion, choisissent des effets picturaux corollaires à cet imaginaire. Les moyens plastiques utilisés s'inscrivent ainsi en marge des qualités traditionnellement reconnues à la peinture du moins, si l'on se base sur les théories de l'art de peindre de la fin du Moyen Âge. Nuages, montagnes, forêts et cavernes se trouvent façonnés tantôt par des lignes rendant grâce à l'inventivité formelle de l'artiste courbes, spirales, images doubles -, tantôt par des manières de rendre imperceptible le visible - ombres, tâches, coulures. En un mot, soit la perspective spatiale se perd, soit la figuration disparaît. Cet abandon 
progressif de structure permet au peintre de déposer des indices de son processus créatif à travers la trace de son art en cours de (dé)construction. La nécessité d'ordonner le monde laisse dès lors place à un geste créateur, comme s'il n'était plus question pour la peinture d'émuler la nature seulement pour ses propriétés organisatrices ou créatives. Désormais l'art de peindre rivalise aussi avec la puissance matricielle du monde créé puisque l'un comme l'autre renouvellent de manière autonome leur propre visibilité. 


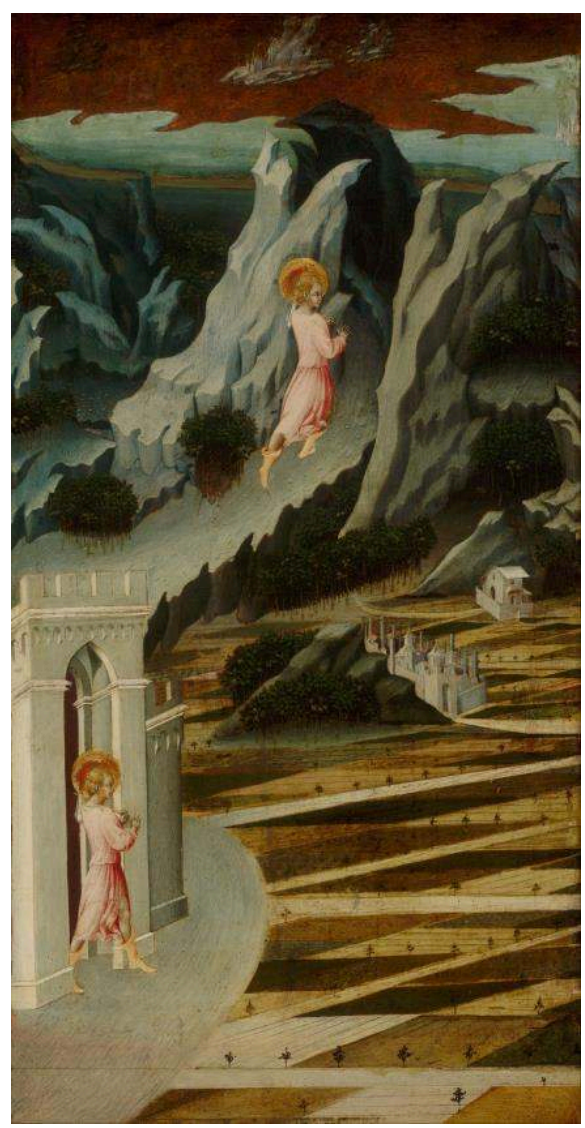

Fig. 1. Giovanni di PAolo, SAint Jean-Baptiste se Retirant Au desert, 1455-1460. Detrempe SUR PANNEAU, 68,5 x 39,5 CM. ARt InStitute, ChicAgo.

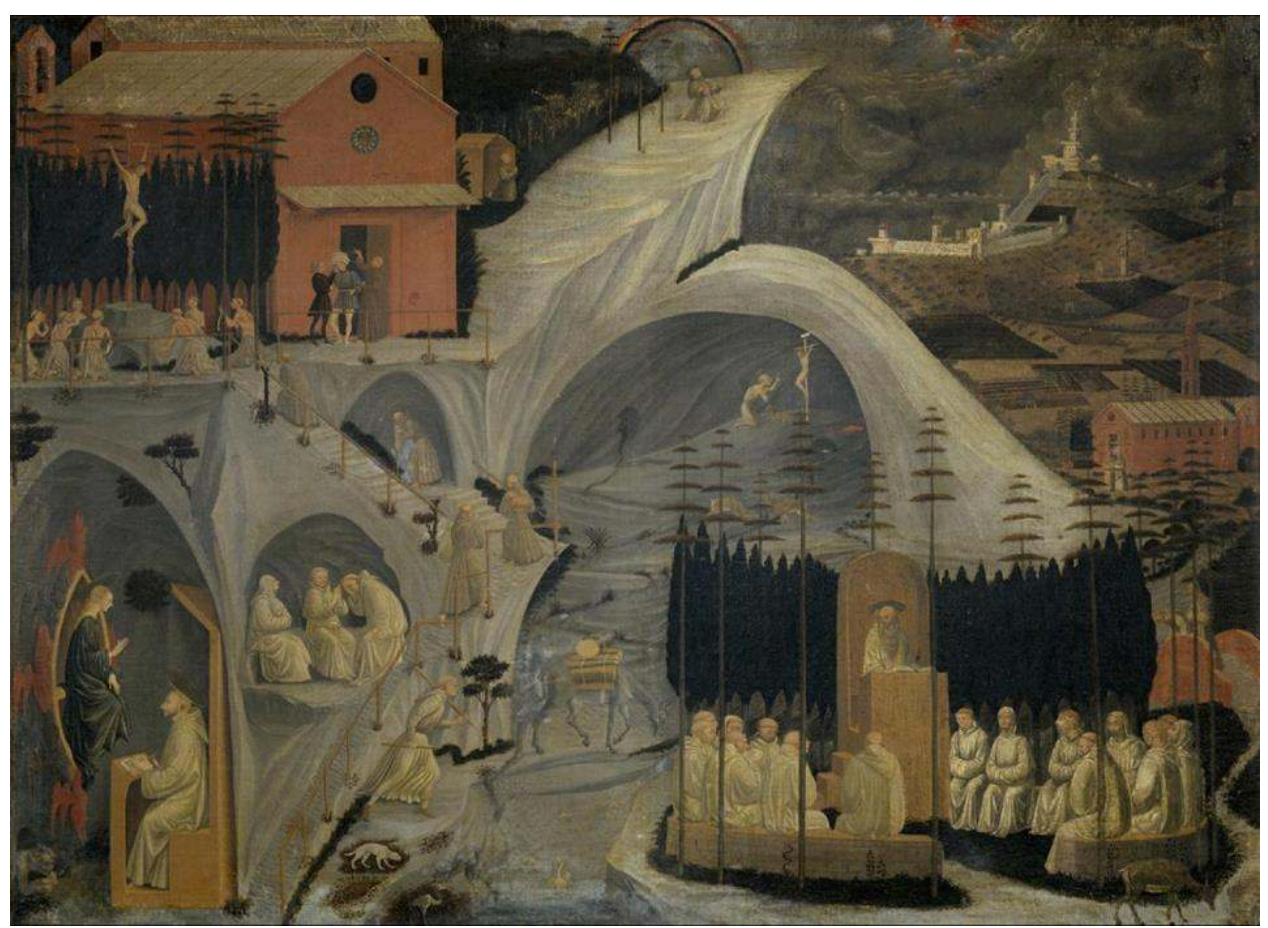

Fig. 2. Paolo Uccello (attribue A), Scenes de legendes monastiques, v.1470. Peinture sur TOILE, 81 × 110 CM. GalleRIA DelL' AcCAdemia, Florence. 


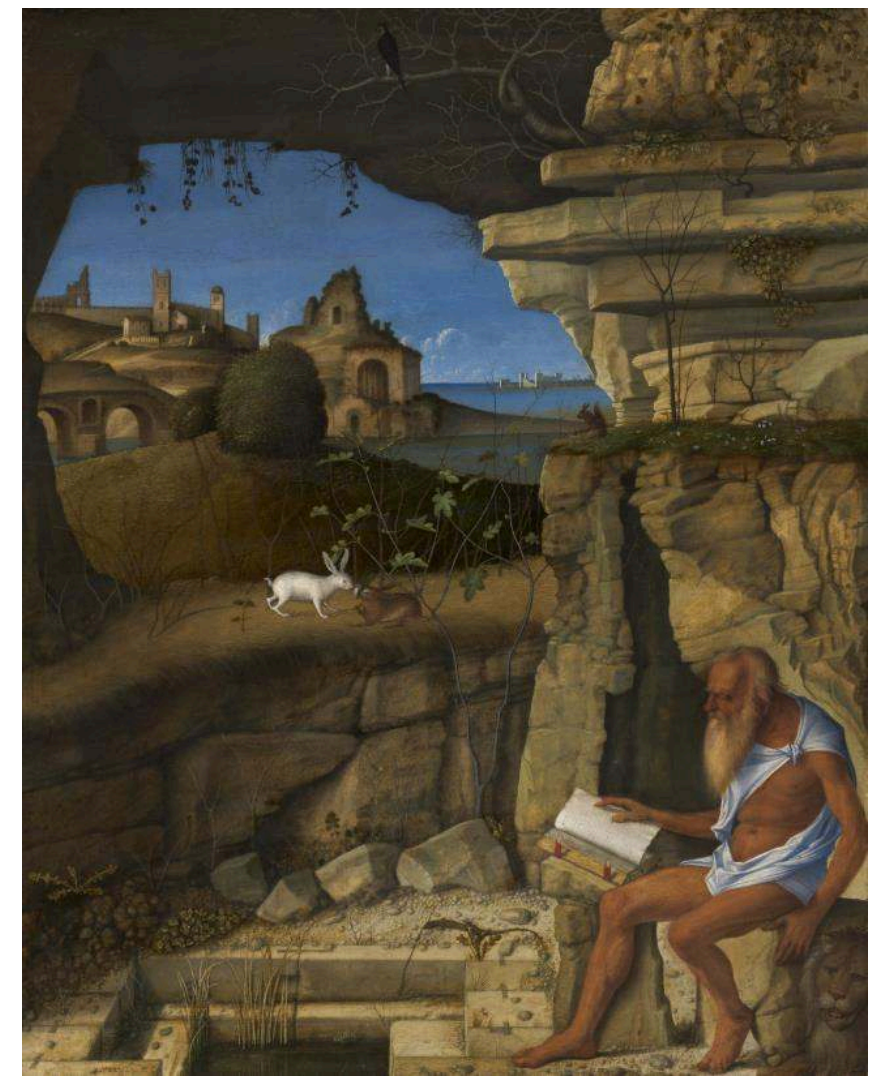

Fig. 3. Giovanni Bellini, Saint Jerome dans le desert, 1505. Huile sur panneau, $47 \mathrm{X}$ 37,5 cm. Samuel H. Kress Collection, National Gallery of Art, Washington. 